\title{
STUDY OF NERVE FIBER TRACKING METHODOLOGIES USING DIFFUSION TENSOR MAGNETIC RESONANCE IMAGING
}

\author{
M. M. Tawfik ${ }^{1}$, Y. M. Kadah ${ }^{1}$ \\ ${ }^{1}$ Institute of Biomedical Engineering, Cairo University, Giza, Egypt \\ e-mail: marwa.tawfik@k-space.org
}

\begin{abstract}
Neural connectivity studies are extremely important for interpreting functional magnetic resonance imaging (FMRI) data and brain in vivo studies. By assuming that the largest principal axis of the diffusion tensor aligns with the predominant fiber orientation in an MRI voxel, we can obtain 2D or 3D vector fields that represent the fiber orientation at each voxel. An algorithm was developed for tracking brain white matter fibers using diffusion tensor magnetic resonance imaging (DT- MRI), which is the only approach now to non-invasively study the architecture of white matter tracts.

Keywords- diffusion, tractography, white matter, fractional anisotropy, slope.
\end{abstract}

\section{I- INTRODUCTION}

Diffusion tensor magnetic resonance imaging (DT-MRI) is the first noninvasive in vivo imaging modality with the potential to generate fiber tract trajectories in soft fibrous tissues, such as the brain white matter (WM) [1]. By assuming that the largest principal axis of the diffusion tensor aligns with the predominant fiber orientation in an MRI voxel, we can obtain 2D or 3D vector fields that represent the fiber orientation at each voxel, Fig. (1). The 3D reconstruction of tract trajectories, or tractography, is a natural extension of such vector fields [2].

The simplest tracking approach, proposed by mori et. al., shown in Fig. (2), is to:

1- Define starting region.

2- Define seed region.

3- Follow the primary eigenvector.

The tracking is terminated when:

1- Entering isotropic region, gray matter area.

2- Angle between two vectors is greater than a set threshold [2, 3].

\section{II-METHODOLOGY}

a-Single tensor tracking

a-1 Problem Formulation

Mori's algorithm was applied to a WM area from real data provided by collaboration with Emory University/ Georgia Tech shown in Fig.(3). When the algorithm was applied to this area,

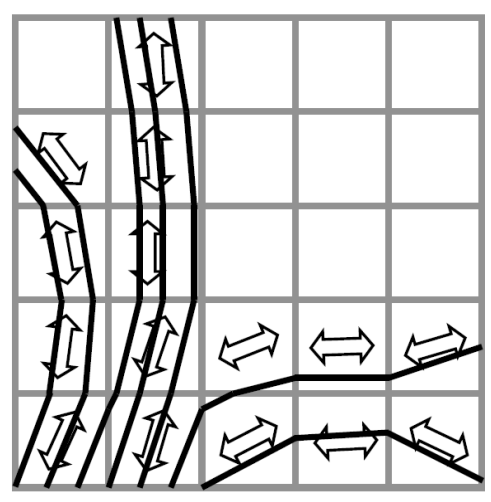

Fig.1. Schematic of the tracking algorithm. Each track follows the direction of greatest diffusion (double-headed arrows) until it hits the neighboring voxel, at which point it follows the new direction.

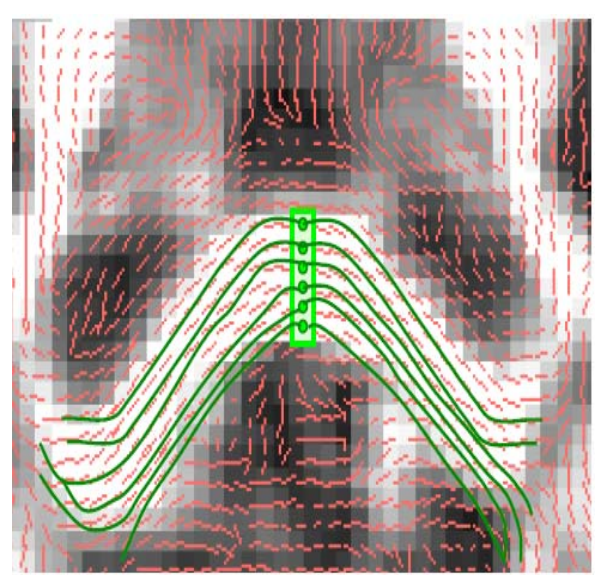

Fig. 2. Mori's algorithm for single-tensor tracking.

a problem was encountered, is that the tract exhibited sharp turns, Fig. (4).

The termination criteria used were:

- Fractional anisotropy (FA) index $<0.1$.

- $\quad$ Angle between two vectors to be connected $>45$. 
Referring to those results, it was a must to look for other tracking criteria in order to smooth the resulting tracts. The criterion added was the tensor angle (slope), which is different from the condition of the angle between tensors. Fig. (5).

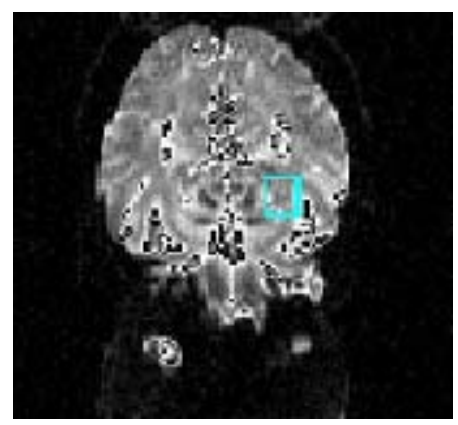

Fig. 3. The selected ROI

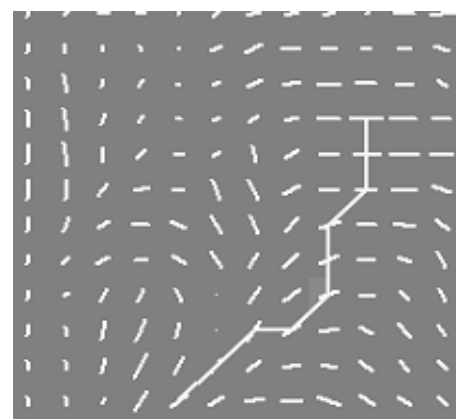

Fig. 4. Tract got from applying Mori’s algorithm on part of the ROI in Fig. 3

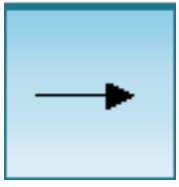

(a)
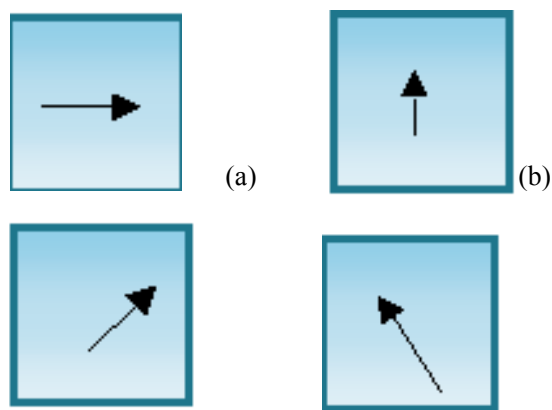

(c)

(d)

Fig.5. Different slopes of eigenvectors. a) A vector pointing to $\mathrm{X}$ direction. b) a vector pointing to $\mathrm{Y}$ direction. $\mathrm{c}, \mathrm{d}$ ) diagonal.

\section{a-2 Methods}

When drawing the velocity plot of the two tensors, we obtain two components, $\mathrm{X}$ and $\mathrm{Y}$.

The tracking algorithm implemented was as follows:

If $\operatorname{abs}(\mathrm{x})$ component is greater than twice $\mathrm{abs}(\mathrm{y})$ component; it was supposed that the seed point is pointing to $\mathrm{X}$ direction, Fig. (5.a);

Firstly, tracking was implemented in positive $\mathrm{X}$ direction, satisfying the criteria of:
1 - FA $>0.1$.

2-Angle between the two connected vectors is less than 45 .

3- The slope of the vector is in $\mathrm{X}$ direction.

Then, the same steps were applied for tracking in the negative direction.

If the $\mathrm{y}$ component of the seed is greater than twice the $\mathrm{x}$ component, then it is supposed that the seed's slope is in Y direction and we track in the Y direction, Fig. (5b).

Firstly, tracking was implemented in the positive $\mathrm{Y}$ direction, satisfying the angle, FA, and slope conditions. Tracking was terminated when one of the previous conditions was violated.

If the pixel with the highest FA did not satisfy angle or slope condition, check the pixel with lower FA.

Loop in order to get a complete tract.

Track in the reverse direction applying the same steps above.

If the seed's $\mathrm{x}$ and $\mathrm{y}$ components did not satisfy either of the conditions above, then:

For vectors with positive slope shown in Fig. (6), the following algorithm was implemented:

1- Track in the positive direction, satisfying the criteria of FA, angle between the vectors, and the slope.

2- The end seed got from the previous step was compared to neighbors, and connected to the pixel with the highest FA, to guarantee entering the most anisotropic region.

3- Loop in order to get a complete tract.

4- Track in the reverse direction with the same steps.

For vectors with negative slope, Fig. (7), the following algorithm was implemented:

1- Track in the negative direction, with satisfying the criteria of FA, angle, and slope.

2- The end seed got from the previous step is compared to neighbors and connected to the pixel with the highest FA provided that this neighbor satisfies the angle Condition.

3- Loop in order to get a complete tract.

4- Track in the reverse direction with the same algorithm above.

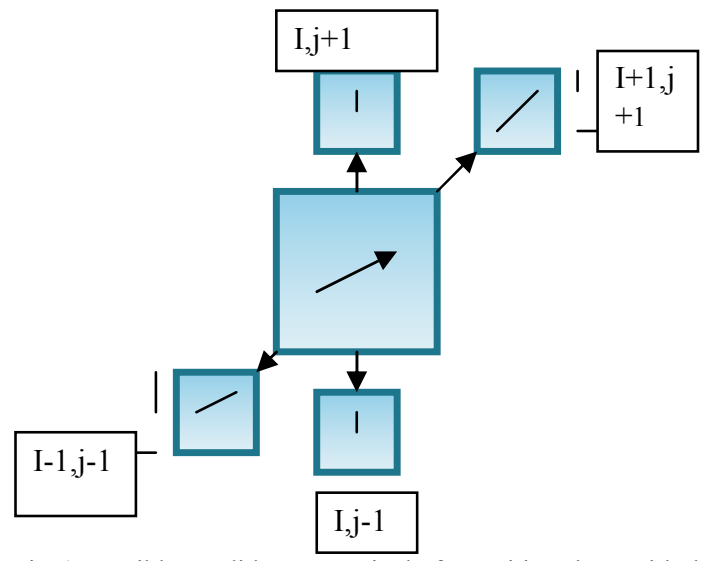

Fig.6. Possible candidates to a pixel of a positive slope with the plotted orientation. The other 4 neighbors are discarded to guarantee smooth fibers. 


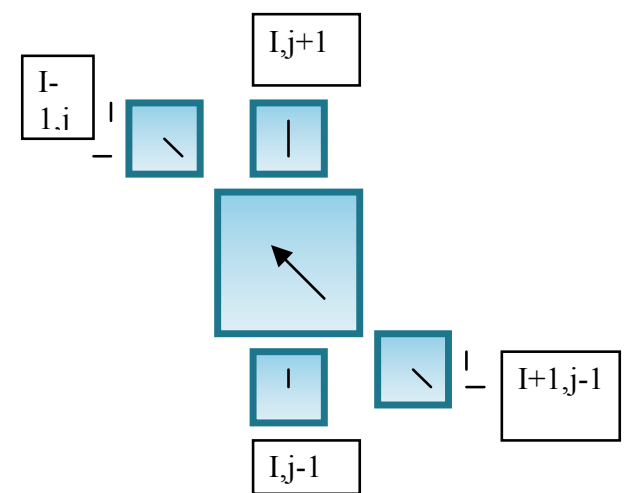

Fig. 7. Possible candidates to a pixel of a negative slope with the plotted orientation. The other 4 neighbors are discarded to guarantee smooth fibers.

\section{b-Multi- tensor tracking:}

Recent studies have shown that the diffusional signal decay in human brain is non-monoexponential and may be described in terms of compartmentalized water fractions, equation (1) [4].

$$
E\left(q_{k}\right)=\sum_{j} f_{j} \exp \left(-q_{k}{ }^{T} D_{j} q_{k} \tau\right)
$$

Where $\mathrm{fj}$ is the apparent volume fraction of the voxel with diffusion tensor $\mathrm{Dj}$. The objective then is to find the set of $\mathrm{n}$ tensors $\{\mathrm{Dj}\}$ and corresponding volume fractions $\{\mathrm{fj}\}$ that best explain the observed diffusion signal.

Simulations along with datasets were used to track the fibers in multiple tensors. This work followed the work of Tuch et. al. [5], Fig. (8), who proposed a model for solving Eq. (1) using some physiological constraints on the eigenvalues, instead of using the traditional method for solving Gaussian mixture problems of this type.

First of all, using the results obtained from single tensor tracking, one tensor for each pixel was obtained. From these results, we have one tensor of the two used for multi- tensor tracking.
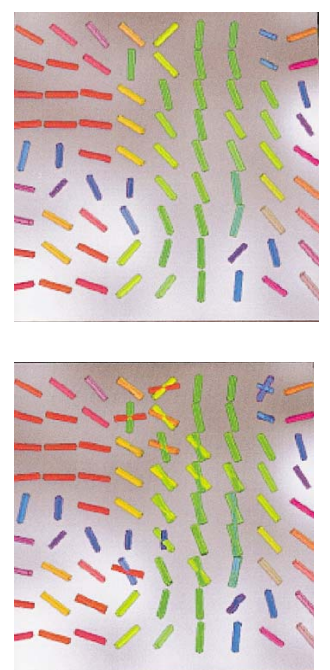

Fig.8. Comparison of the principal eigenvector fields from the (a) singletensor and (b) two-tensor fits to diffusion signal from the forceps minor, Tuch et. al.
The other tensor of each pixel was generated as follows: 1- In each pixel, generate a tensor $\mathrm{D}_{2}$ such that:

$\mathrm{D}_{2}=[\operatorname{Transpose}(\mathrm{R})] \times\left[\left[\lambda_{1}, 0,0\right],\left[0, \lambda_{2}, 0\right],\left[0,0, \lambda_{3}\right]\right] \times[\mathrm{R}]$, where $\mathrm{R}$ is the rotation matrix and $\lambda_{1}=1.5, \lambda_{2}=0.4, \lambda_{3}=0.4$, and using different angles of rotation, were chosen randomly between $10^{\circ}$ and $60^{\circ}$.

- Rotation matrix used:

[[cos $(\theta),-\sin (\theta), 0],[\sin (\theta), \cos (\theta), 0],[0,0,1]][4]$.

2- Partial volumes, $f_{\mathrm{i}}$, were generated so that they add one in each pixel, Eq. (1).

We had two tensors in each pixel then, Fig. (9). The tracking procedure was implemented as follows:

1. Choose a seed point, one of the two components of a pixel.

2. According to its slope, select which of the eight neighbors could be connected to the current seed.

3. Compare the partial volume of the current point to the partial volume of the candidate point. The next point on the tract should satisfy the following criteria:

- PV (current seed) - $0.1=<$ PV (candidate) $<=$ PV (current seed) +0.1 ; this is to achieve mass balance.

- Angle between two pixels $<=45$.

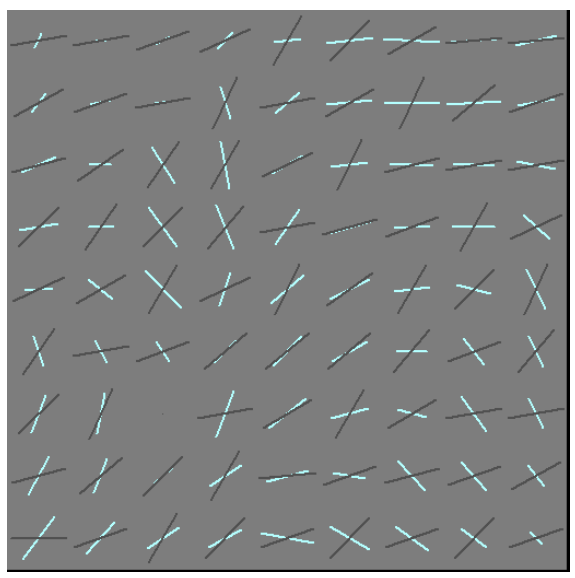

Fig.9. The generated multiple tensors.

\section{III- RESULTS and DISCUSSION \\ a- Single tensor Tracking}

Several individual tracts were got from tracking in the $\mathrm{X}$, $\mathrm{Y}$, and the diagonal directions.

Figs. (10), (11), and (12) show different tracts got from the tracking procedure implemented. 


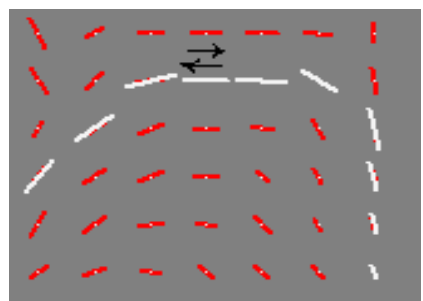

Fig.10. results from tracking in the $\mathrm{X}$ direction.

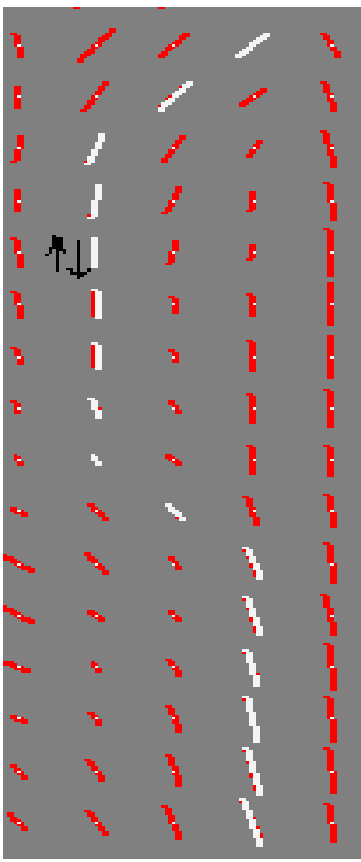

Fig. 11. Tracking results in $\mathrm{Y}$ direction.

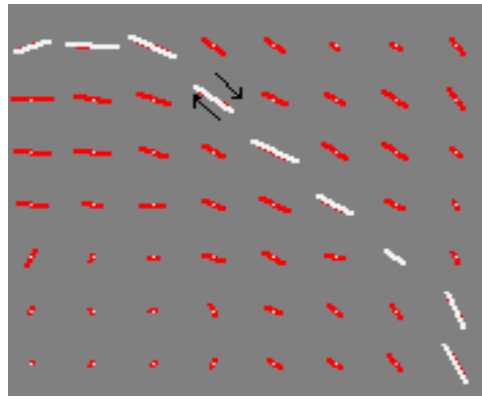

Fig. 12. Tracking in diagonal direction

Fig. (13) shows all the tracts obtained from the selected ROI shown in Fig. (3).

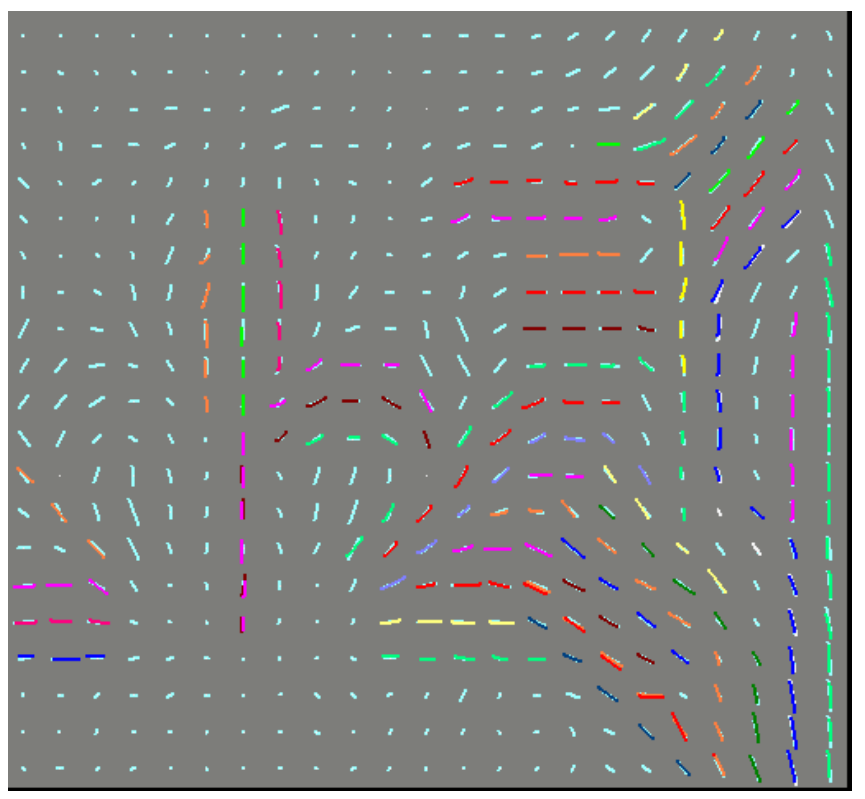

Fig.13. Tracts of the selected ROI, each with a specific color.

b- Multi-tensor Tracking

Fig. (14) shows 2 crossing fibers resulted form implementing the developed algorithm.

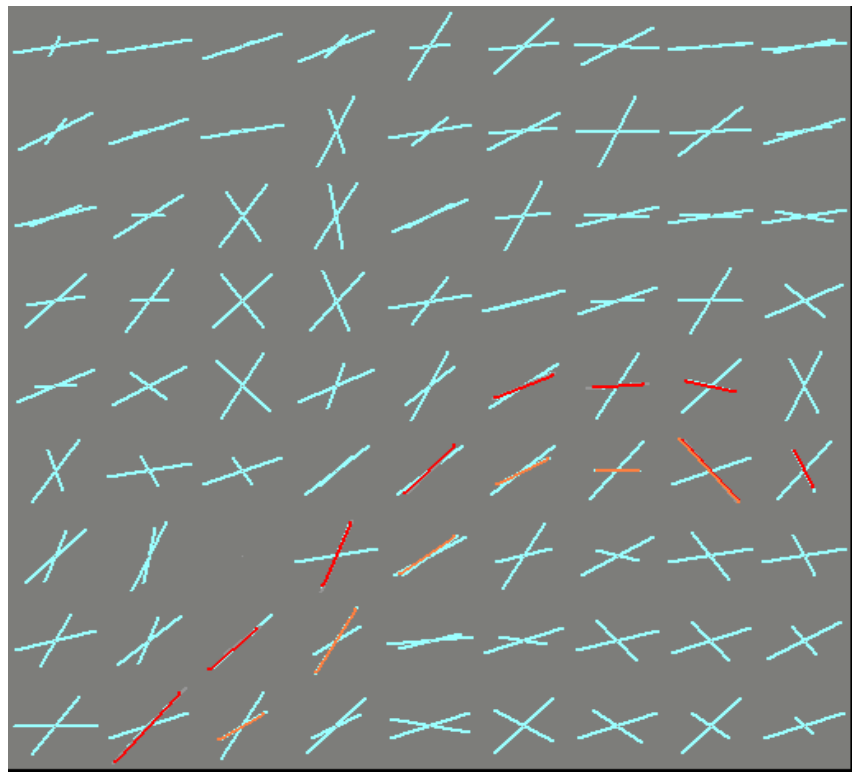

Fig. 14. Two crossing fibers resulted from multi-tensor tracking. 


\section{IV- Conclusions}

The proposed method of tracking solved the problem of sharp turns, but in general Tractography needs high signal to noise ratio (SNR) as the higher the SNR of the image, the smoother the tracts obtained.

Neural connectivity studies are extremely important for interpreting FMRI data and have a great impact on brain in vivo studies.

An algorithm was developed for tracking fibers using DTMRI, which is the only approach now to non-invasively study the architecture of white matter tracts. The algorithm depended heavily on the tensor slope.

Simulations were used along with the dataset for the problem of multi- tensor tracking.

\section{References}

[1] J. Zhang, H. Ji, N. Kang, N. Cao, "Fiber Tractography in Diffusion Tensor Magnetic Resonance Imaging: A survey and beyond", Technical Report No. 437-05, Department of Computer Science, University of Kentucky, Lexington, KY, 2005.

[2] S. Mori, P.C.M. Van Zijl, "Fiber Tacking: principles and strategies- a technical review", NMR Biomed., vol. 15, pp. 468-480, 2002.

[3] H. Jiang, P.C.M. Van Zijl, J. Kim, G. D. Pearlson, S. Mori, "DTI Studio: resource program for diffusion tensor computation and fiber bundle tracking ", Computer Methods and Programs in Biomedicine, vol.8, pp.106-116, 2006.

[4] C. A. Clark, M. Hedehus, M. E. Moseley, "In Vivo Mapping of the Fast and Slow Diffusion Tensors in Human Brain”, Magn. Reson. Med., vol.47, pp. 623- 628, 2002.

[5] D. S. Tuch, T. G. Reese, M. R. Wiegell, N. Makris, J. W. Belliveau, V. J. Wedeen, "High Angular Resolution Diffusion Imaging Reveals Intravoxel White Matter Fiber Heterogeneity", Magn. Reson. Med., vol.48, pp. 577-582, 2002. 